\title{
A TOPOLOGICAL PERSISTENCE THEOREM FOR NORMALLY HYPERBOLIC MANIFOLDS VIA THE CONLEY INDEX
}

\author{
ANDREAS FLOER
}

\begin{abstract}
We prove that the cohomology ring of a normally hyperbolic manifold of a diffeomorphism $f$ persists under perturbation of $f$. We do not make any quantitative assumptions on the expansion and contraction rates of $D f$ on the normal and the tangent bundles of $N$.
\end{abstract}

\section{INTRODUCTION}

In this paper, we shall apply C. Conley's homotopy index theory for invariant sets of flows to discrete dynamical systems. In particular, we prove a homotopy version of the persistence theorem for normally hyperbolic invariant manifolds.

Let $M$ be a smooth i.e. $\mathscr{C}^{2}$ manifold and let $f: M \rightarrow M$ be a diffeomorphism which restricts to a diffeomorphism of a compact $\mathscr{C}^{2}$ submanifold $N$ onto itself. One calls $N$ normally hyperbolic with respect to $f$ if it satisfies a certain nondegeneracy condition on a normal bundle of $N$ in $M$. In This paper, we will use the following definition:

Definition 1. $N$ is called normally hyperbolic with respect to $f$ if the tangent bundle of $M$ over $N$ splits into smooth subbundles

$$
\left.T M\right|_{N}=E^{+} \oplus T N \oplus E^{-}
$$

which are invariant under $D f: T M \rightarrow T M$ such that $D f$ contracts $E^{-}$and expands $E^{+}$. Here we say that a vector bundle isomorphism $\psi: E \rightarrow E$ contracts $E$ if for every $\xi \in E$, the sequence $\psi^{n} \xi$ converges to the zero section of $E$. We say that $\psi$ expands $E$ if $\psi^{-1}$ contracts $E$.

N. Fenichel [2] examined the question under which conditions a normally hyperbolic manifold persists under small perturbations of $f$. In fact, he proves that if $D f$ contracts $E^{-}$and expands $E^{+}$stronger than any vector in $T N$, then every $\mathscr{C}^{1}$-small perturbation of $f$ has a normally hyperbolic manifold $\mathscr{C}^{1}$-diffeomorphic to $N$ (see also [6, Theorem 4.1] for more persisting properties of $N$ ). Here, the expansion and contraction rates have to be measured by some metric on $M$. In order to illustrate the importance of this quantitative

Received by the editors April 2, 1987 and, in revised form, November 8, 1988.

1980 Mathematics Subject Classification (1985 Revision). Primary 58F10; Secondary 58F14. 
hypothesis, consider on $N$ an attracting fixed point such that the rate of approach in the direction of $T N$ is greater than in the normal direction. At such a point, a cusp may develop under perturbation (see [2, 8, and 4, pp. 239, 251]). There are also examples (see Jarnik and Kurzweil, [7]), where a normally hyperbolic manifold changes under arbitrarily small perturbations into an invariant set which is not even a topological manifold. In this paper, we shall prove that if $N$ is normally hyperbolic in the sense of Definition 2, i.e. without any additional quantitative conditions, then the cohomology of $N$ persists under $\mathscr{C}^{0}$ small perturbations of $f$ :

Theorem 1. Let $f: M \rightarrow M$ be a diffeomorphism and let $N$ be a smooth compact normally hyperbolic invariant submanifold with respect to $f$. Let $\left\{f_{\lambda}\right\}_{\lambda \in \mathbb{R}}$ be a family of homeomorphisms of $M$, continuous in the compact-open topology, with $f_{0}=f$. Then for $\lambda$ small enough, $f_{\lambda}$ has an isolated invariant set $T_{\lambda}$ whose cohomology ring contains $H^{*}(N)$ as a subring. In fact, for every neighborhood $U$ admitting a retraction $r: U \rightarrow N$, we have $T_{\lambda} \subset U$ for $\lambda$ small enough, and

$$
\left(r \mid T_{\lambda}^{*}\right): H^{*}(N) \rightarrow H^{*}\left(T_{\lambda}\right)
$$

is injective. Here, coefficients are arbitrary if $N$ and $E^{+}$are oriented and $f \mid N$ and $\left.D f\right|_{E^{+}}$are orientation preserving and in $\mathbb{Z}_{2}$ otherwise.

The notion of an isolated invariant set will be defined in the following section.

In analogy to [3, Theorem 2], one also has a global persistence result for certain normally hyperbolic manifolds $N$, which does not assume that the perturbation is small. Instead, one has to assume, in addition, that $N$ is a retract of $M$ by a retraction which commutes with $f$ up to homotopy. This means that there exists a continuous map

$$
r: M \rightarrow N
$$

such that $r \mid N$ is homotopic to the identity and so that $f \mid N \circ r$ and $r \circ f$ are homotopic as maps from $M$ to $N$.

Theorem 2. Let $N \subset M$ be a compact normally hyperbolic invariant manifold of the diffeomorphism $f$ and let $r: M \rightarrow N$ be a retraction commuting with $f$ up to homotopy. Then for every invariant set $T$ of a homeomorphism $f^{\prime}$ which is related to $(N, f)$ by continuation, $r$ induces injective homomorphisms

$$
(r \mid T)^{*}: H^{*}(N) \rightarrow H^{*}(T)
$$

with coefficients as in Theorem 1.

The notion of continuation will be defined in the following section. The proof of Theorems 1 and 2 uses the index theory for topological flows developed by C. Conley (see [1]). By a suspension procedure ( $\$ 2)$ we construct a flow on a bundle $M_{f}$ over $S^{1}$ with fibre $M$ whose time 1 map equals $f$. In $\S 3$, we show that a normally hyperbolic submanifold $N$ of $M$ for $f$ corresponds to a 
normally hyperbolic submanifold $N_{f}$ of $M_{f}$ of the flow. In this situation, we can apply Theorem 2 of [3] to continue $N_{f}$ under a perturbation of the flow. In $\S 4$, we show that this proves the perturbation result for the map $f$ on $N$.

\section{MAPS AND FLOWS}

We will denote by $\mathscr{F}(M)$ the space of all homeomorphisms from a locally compact Hausdorff space $M$ onto itself. In some applications, one also considers an operation $\rho$ of a compact topological group $G$ on $M$, i.e. a continuous map

$$
\rho: G \times M \rightarrow M, \quad(g, x) \mapsto g y
$$

such that $(g \circ h) x=g(h x)$. In such a situation, we may restrict ourselves to the subset $\mathscr{F}_{\rho} \subset \mathscr{F}(M)$ of homeomorphisms commuting with $\rho(g)$ for all $g \in G$, i.e. of equivariant homeomorphisms. Let $\mathscr{F}_{\rho}$ be equipped with any Hausdorff topology so that the inverse map

$$
\mathscr{F} \rightarrow \mathscr{F}: f \mapsto f^{-1}
$$

is continuous, for example, we can take the compact open topology. In analogy to [3, Definition 1], we can introduce the notion of an isolated invariant set

Definition 2.1. For any $\rho$-invariant subset $U$ of $M$, and for $f \in \mathscr{F}_{\rho}$, define

$$
\begin{aligned}
& T_{-}^{f}(U)=\left\{x \in U \mid f^{n}(x) \subset U \text { for all } n \geq 0\right\}, \\
& T_{+}^{f}(U)=\bigcap_{n \geq 0} f^{n}(U) .
\end{aligned}
$$

The maximal invariant set is defined by

$$
T^{f}(U)=T_{-}^{f}(U) \cap T_{+}^{f}(U) .
$$

We call $U$ isolating, if the closure of $T^{f}(U)$ is contained in the interior of $U$. In this case, $T^{f}(U)$ is called an isolated invariant set.

Next we define the notion of continuation. Define the set

$$
\begin{array}{r}
\mathscr{T}=\left\{(T, f) \mid f=\mathscr{F}_{\rho} \text { and } T\right. \text { is a nonempty compact } \\
\text { isolated invariant set of } f \text { in } M\} .
\end{array}
$$

On $\mathscr{T}$, consider the topology generated by the open sets

$$
\begin{aligned}
& \theta_{U}=\{(T, f) \mid U \text { is isolated with respect to } f \text { with } \\
& \text { nonempty maximal invariant set } \left.T=T^{f}(U)\right\},
\end{aligned}
$$

where $U$ is any open ( $\rho$-invariant) subset of $M$.

Definition 2.2. Two elements $(T, f)$ and $\left(T^{\prime}, f\right)$ of $\mathscr{T}$ are called related by continuation, if they are connected to $\mathscr{T}$ by a continuous path.

We want to define a topological index for isolated invariant sets of maps $f$ on $M$ which is invariant under continuation, i.e. which only depends on the 
path components in $\mathscr{T}$. Therefore, we use the index theory for flows developed by C. Conley. A flow on a topological space $\Gamma$ is defined as a continuous map

$$
\chi: \Gamma \times \mathbb{R}_{+} \supset U_{\Gamma} \rightarrow \Gamma:(x, t) \rightarrow \chi(x, t)=x \cdot t,
$$

where $U_{\Gamma}$ is a neighborhood of $\Gamma \times\{0\}$ in $\Gamma \times \mathbb{R}_{+}$with the following property: If $(x, t)$ and $(x \cdot t, x) \in U_{\Gamma}$, then $(x, t+s) \in U_{\Gamma}$ and

$$
(x \cdot t) \cdot s=x \cdot(t+s) .
$$

Again, we can assume that the flow is equivariant with respect to some operation $\rho$ of a compact group $G$ on $\Gamma$, i.e. that $U_{\Gamma}$ is $\rho$-invariant and that

$$
\rho(x) \cdot t=\rho(x \cdot t) \quad \text { for }(x, t) \in U_{\Gamma} .
$$

Moreover, we can define isolated invariant sets (see, for example [3, Definition 1]),

Definition 2.3. For any ( $\rho$-invariant) subset $U$ of $\Gamma$, define

$$
\begin{aligned}
U_{-\infty} & =\left\{x \in U \mid x \cdot \mathbb{R}_{+} \subset U\right\}, \\
U_{\infty} & =\bigcap_{t \geq 0} U \cdot t
\end{aligned}
$$

and the maximal invariant set

$$
S(U)=U_{\infty} \cap U_{-\infty} .
$$

We are concerned with the following family of flows:

Definition 2.4. Consider the quotient

$$
M_{\mathscr{F}}=p\left(M \times \mathscr{F}_{\rho} \times(-1,1)\right),
$$

where $p$ is the projection map corresponding to the equivalence relation generated by

$$
(x, f, t-1) \sim(f(x), f, t)
$$

for $t \in(0,1)$. On $M_{\mathscr{F}}$, consider the flow $\chi$ defined by the map

$$
\chi^{\tau}(x, f, t)=(x, f, t-\tau)
$$

for $\tau, t \in[0,1)$.

We can define an operator $\rho$ on $M_{\mathscr{F}}$ commuting with the flow by

$$
\rho(g)(x, f, t)=(\rho(g) x, f, t) .
$$

Clearly, the flow $\chi$ on $M_{\mathscr{F}}$ restricts to a continuous flow $\chi_{f}$ on every leaf

$$
M_{f}:=p_{f}(M \times(-1,1)),
$$

where $p_{f}$ is the restriction of $p$ to $M \times(-1,1) \cong M \times\{f\} \times(-1,1)$. The flow $\chi_{f}$ is also called the suspension of the map $f$. 
Proposition 1. The flow invariant map

$$
M_{\mathscr{F}} \rightarrow \mathscr{F}(M):(x, f, t) \mapsto f
$$

defined a ( $\rho$-invariant) local product parametrization in the sense of $[3$, Definition 3].

Proof. It suffices to show that the map

$$
\begin{gathered}
M \times \mathscr{F}_{\rho} \times \mathbb{R} \rightarrow M \times \mathscr{F}_{\rho} \times \mathbb{R}, \\
(x, f, t) \mapsto(f(x), f, t+1)
\end{gathered}
$$

is a homeomorphism. In fact, in this case, we have $M_{\mathscr{F}}=\left(M \times \mathscr{F}_{\rho} \times \mathbb{R}\right) / \mathbb{Z}$, where the operation of $\mathbb{Z}$ is generated by the map (2.19). Since $M \times \mathscr{F}_{\rho} \times \mathbb{R}$ is a product space, this proves that $M$ has the required local product structure.

Obviously, the map (2.19) is bijective and continuous, $f \in \mathscr{F}_{\rho}$ is a homeomorphism and the evaluation map $\mathscr{F}_{\rho} \times M \rightarrow M$ is continuous. But also the inverse

$$
(x, f, t) \mapsto\left(f^{-1}(x), f, t-1\right),
$$

is continuous, since the map (2.1) was assumed to be continuous on $\mathscr{F}_{\rho}$.

We now can define the notion of continuation in $M$. Following [1, Section $4]$ and [3, Section 2], we consider the set

$(2.21) \cdot \mathscr{S}=\left\{(S, f) \mid f \in \mathscr{F}_{\rho}(M)\right.$ and $S$ is a nonempty compact isolated invariant (and $\rho$-invariant) set in $\left.M_{f}\right\}$.

The topology is generated by the open sets

$$
\Sigma_{U}=\left\{(S, f) \mid U \cap M^{f} \text { is isolating in } M_{f} \text { with maximal invariant set } S\right\},
$$

where $U$ is any open ( $\rho$-invariant) subset of $M_{\mathscr{F}}$.

Proposition 2. The map

$$
\Psi: \mathscr{T} \rightarrow \mathscr{S}:(T, f) \mapsto\left(T_{f}, f\right)
$$

is a homeomorphism.

Proof. First, note that every nonempty invariant set of $\chi_{f}$ on $M_{f}$ is of the form $T_{f}$, where $T$ is an invariant set of $f$. We now show that for every open set $U \subset M$ and for every $f \in \mathscr{F}$, the set $U_{f}$ is isolating for the flow $\chi_{f}$ if and only if $U$ is isolating for $f$. In fact, it is easy to see that $x \in T_{f}^{+}(U)$ if and only if $p_{f}(x, t) \in U_{\infty}^{f}$ for all $t \in(-1,1)$. Similarly, $x \in \mathscr{T}^{-}(U, f)$ if and only if $p_{f}(x, t) \in U_{-\infty}^{f}$ for all $t-\in I$.

This shows that $T$ is isolated in $U$ if and only if $T^{f}$ is isolated in $U^{f}$. Moreover, if $\widetilde{U}$ is any isolating neighborhood of $T^{f}$ in $M_{f}$, then it contains a neighborhood of the form $U^{f}$ for some neighborhood $U$ of $T$ in $M$. Hence the map $\Psi$ of Proposition 2 is bijective. 
In order to show that it is also a homeomorphism, note that the topology of $\mathscr{F} \times M$ is generated by sets $U=U_{m} \times U_{\mathscr{F}}$, where $U_{\mathscr{F}}$ is open in $\mathscr{F}$ and $U_{M}$ is open in $M$. Similarly, the topology of $M_{\mathscr{F}}$ is generated by sets $p\left(U_{M} \times U_{\mathscr{F}} \times I\right)$, where, in addition, $I \subset(-1,1)$ is open. However, since we are only interested in neighborhoods $U$ of $(S, f) \in \mathscr{S}$ for which $S=S^{f}(U)$ is nonempty, we can restrict ourselves to those open sets where $I=(-1,1)$. Then by the above,

$$
\Sigma_{p\left(U_{M} \times U_{\mathscr{F}} \times I\right)}=\left\{\left(S^{f}, f\right) \mid(S, f) \in \Theta_{U_{M} \times U_{\mathscr{F}}}\right\}=\Psi\left(\theta\left(U_{M} \times U_{\mathscr{F}}\right)\right),
$$

which completes the proof of Proposition 2.

We can use the map $\Psi$ and the Conley index for flows (see [1 and 3]) to define a topological index on $T$.

Definition 2.5. For $(T, f) \in \mathscr{T}$, define

$$
I_{\rho}^{*}(T, f):=H_{\rho}^{*}(X, A)
$$

where $(X, A)$ is any ( $\rho$-invariant) index part for $\mathscr{T}_{f} \subset M_{f}$ and $H_{\rho}^{*}$ denotes the equivariant Alexander-Spanier cohomology with values in some ring with unit. Moreover, with $\pi: M_{f} \rightarrow S^{1}$ given by $\pi(x, t)=t$ and for the standard generator $e$ of $H^{1}\left(S^{1}\right)$, define

$$
\theta_{(T, f)}: I_{\rho}^{*}(T, f) \rightarrow I_{\rho}^{*}(T, f): \alpha \mapsto \alpha \cup\left(\left.\pi\right|_{x}\right)^{*} e .
$$

The continuation invariance of the index $I_{\rho}=\left(I_{\rho}^{*}, \theta\right)$ on $\mathscr{T}$ then follows immediately from Proposition 2 and from Theorem 1 of [3].

Theorem 3. $I_{\rho}^{*}(T, f)$ does not depend on the choice of the index pair $(X, A)$. Moreover, if $(T, f)$ is related to $\left(T^{\prime}, f^{\prime}\right)$ by continuation, then there exists an isomorphism $i: I_{\rho}^{*}(T, f) \rightarrow I_{\rho}^{*}\left(T^{\prime}, f^{\prime}\right)$ such that

$$
\theta_{(T, f)} \circ i=i \circ \theta_{\left(T^{\prime}, f^{\prime}\right)} \text {. }
$$

\section{SUSPENDING NORMALLY HYPERBOLIC MANIFOLDS}

In this section, we prove the following proposition.

Proposition 3. If $N \subset M$ is a normally hyperbolic invariant submanifold with respect to a smooth map $f$, then $N_{f}$ is a normally hyperbolic invariant submanifold of $M_{f}$ with respect to the suspended flow in the sense of [3, Proposition 1].

Note therefore that, by Definition $1, f$ is in fact a diffeomorphism from some neighborhood $O$ of $N \subset M$ onto its image. Therefore,

$$
O_{f}:=p_{f}(O \times(-1,1)) \subset M_{f}
$$

is a smooth open manifold with a smooth submanifold $N_{f}=p_{f}(N \times(-1,1))$. Now consider on $N_{f}$ the vector bundles

$$
E_{f}^{ \pm}=p_{D f}\left(E^{ \pm} \times(-1,1)\right)
$$


where $p_{D f}$ identifies $(\xi, t-1)$ with $\left(f_{*} \xi, t\right)=(D f \xi, t)$ for $t \in(0,1)$. This is well defined, since $f_{*}$ leaves $E^{ \pm}$invariant. Moreover, (3.2) constitutes a decomposition of the normal bundle of $N_{f}$ in $O_{f}$. The flow on $O_{f}$ is generated by the vector field

$$
\eta:=\left(p_{f}\right)_{*} \frac{\partial}{\partial t}
$$

which is also well defined on $O_{f}$. In order to show that $N_{f}$ satisfies the conditions of Proposition 1 of [3], we have to find a metric $\bar{g}$ on $O_{f}$ so that for every $v \in N$, the linear operator

$$
L: T_{v}(M) \rightarrow T_{v} M: \xi \mapsto \xi \cdot \nabla_{\bar{g}} \eta
$$

leaves the bundles $E^{ \pm} f$ invariant and satisfies

$$
\begin{aligned}
& \langle\xi, L \xi\rangle \geq m|\xi|^{2} \quad \text { for } \xi \in E_{f}^{+}, \\
& \langle\xi, L \xi\rangle \leq-m|\xi|^{2} \quad \text { for } \xi \in E_{f}^{-}
\end{aligned}
$$

for some constant $m>0$. In (3.4), $\xi \cdot \nabla_{\bar{g}}$ denotes the covariant derivative with respect to the metric $\bar{g}$ in the direction of $\xi$.

The first step towards the construction of the metric $\bar{g}$ is

Lemma 3.1. Let $\psi$ be a contracting bundle map on the vector bundle $E$. For any metric $g_{0}$ on $E$, define $g_{k}=\left(f^{*}\right)^{k} g_{0}$ for $K \in \mathbb{N}$. Then the series

$$
g=\sum_{k=0}^{\infty} g_{k}
$$

converges to a metric on $E$ with

$$
|\xi|_{g}^{2}-\left|f_{*} \xi\right|_{g}^{2}=|\xi|_{g_{0}}^{2}
$$

Proof. Set $B_{\alpha}=\left\{\xi \in E^{-} \mid g_{0}(\xi, \xi) \leq g a^{2}\right\}$. Since $B_{1}$ is compact, the contracting property of $D f$ on $E^{-}$implies that there exists an $n \in \mathbb{N}$ with

$$
f_{*}^{n} B_{1} \subset B_{1 / 2} \text {. }
$$

Consequently, we have

$$
\left|f_{*}^{k \cdot n} \xi\right|_{g_{0}} \leq 2^{-k}|\xi|_{g_{0}}
$$

and hence for some $\mathscr{C}$ independent of $x \in N$ :

$$
\left[\left(f^{k \cdot n}\right)^{*} \gamma\right]_{i j} \leq \mathscr{C} 2^{-k} \text {. }
$$

Hence the sum of (3.7) converges and (3.8) holds. Moreover, $g$ is positive as a sum of positive metrics.

We apply Lemma 3.1 to the contracting bundle maps $D f$ on $E^{-}$and $(D f)^{-1}$ on $E^{+}$to obtain metrics $g^{ \pm}$on $E^{ \pm}$with

$$
\langle\xi, \xi\rangle_{g^{ \pm}}-\left\langle f_{*} \xi, f_{*} \xi\right\rangle_{g^{ \pm}}=\mp\langle\xi, \xi\rangle_{g_{0}} .
$$


We can extend these metrics to a Riemannian metric $g$ on $M$ such that $\left\langle\xi^{-}, \xi^{+}\right\rangle_{g}=0$ for $\xi^{ \pm} \in E^{ \pm}$. Now $\bar{g}$ is defined as the unique metric on $M_{f}$ satisfying

$$
\begin{aligned}
& i_{t}^{*} \bar{g}=t g+(1-t) f^{*} g, \\
& |\eta|_{\bar{g}} \equiv 1, \\
& \left\langle\eta, i_{t}^{*} \xi\right\rangle_{\bar{g}}=0 \quad \text { for } \xi \in T M
\end{aligned}
$$

where $i_{t}: M \rightarrow M_{f}$ is given by $i_{t}(x)=p_{f}(x, t)$. Note that $i_{1}=i_{0} \circ f$, so that condition (3.13) is compatible for $t=0,1$.

To calculate the linear operator $L$ of (3.4), note that for any $(x, t) \in M_{f}$ there exists a neighborhood which is naturally isomorphic by the flow $f^{t}$ to $I \times U$, where $I \subset \mathbb{R}$ and $U \subset M$ are open sets. Choose any chart of $M$ at $x$. Let the index $O$ denote the direction of $t$, so that $\eta$ corresponds to the constant vector field $e^{o}$ on the chart. With $i, j$, and $k$ running from $O$ to $\operatorname{dim} M$, the covariant derivative of $e^{o}$ can now be expressed in terms of Christoffel symbols (see [5])

$$
(L \xi)^{i}=\left(\xi \cdot \nabla_{\bar{g}} e^{o}\right)^{i}=\Gamma_{j k} \xi^{k}\left(e^{o}\right)^{j}=\Gamma_{o k}^{i} \xi^{k} .
$$

By [5], we have therefore $\bar{g}_{i j}(L \xi)^{j}=\Gamma_{i, o k} \xi^{k}$ with

$$
\begin{aligned}
T_{o k, j} & =\frac{\partial}{\partial x^{k}} \bar{g}_{j o}+\frac{\partial}{\partial x^{0}} \bar{g}_{j k}-\frac{\partial}{\partial x^{j}} \bar{g}_{o k} \\
& =\frac{\partial}{\partial t}\left(t g_{j k}+(1-t)\left(f^{*} g\right)_{j k}\right) \\
& =\left(g-f^{*} g\right)_{j k},
\end{aligned}
$$

whereas $\Gamma_{o k, j}=0$ for $k=0$ or $j=0$. Hence we have for $\xi, \zeta \in T_{(x, t)} \bar{M}$ :

$$
\langle\xi, L \zeta\rangle_{\bar{g}}=\left\langle\pi_{*} \xi, \pi_{*} \zeta\right\rangle_{g}-\left\langle f_{*} \pi_{*} \xi, f_{*} \pi_{*} \zeta\right\rangle_{g}
$$

where $\pi: U \times I \rightarrow U$ is the projection. This immediately shows that $L$ leaves the mutually orthogonal subspaces $E^{ \pm}$and $T N_{f}$ invariant. Moreover, choosing $\xi=\zeta \in E^{+}$or $E^{-}$and using (3.8) proves (3.5) and (3.6).

\section{Proof of the continuation Results}

In this section we will complete the proof of Theorems 1 and 2. Let us first consider the situation of Theorem 2. Since it can be done without much additional work, we will always assume that $f \in \mathscr{F}_{\rho}$ for some operation $\rho$ of a compact group $G$. Theorem 2 is obtained by considering $G=\{1\}$.

Theorem 4. Let $\rho, M, f, N$ be as in Proposition 3. Let $r: M \rightarrow N$ be a $\rho$-equivariant retraction such that there is a $\rho$-equivariant homotopy between $\left.f\right|_{N} \circ r$ and $r \circ f$. Then if $T$ is a $\rho$-invariant isolated invariant set of a map $f \in \mathscr{F}_{\rho}$ and $\left(T, f^{\prime}\right)$ is related to $(N, f)$ by continuation,

$$
\left(\left.r\right|_{T}\right)^{*}: H_{\rho}^{*}(N) \rightarrow H_{\rho}^{*}(T)
$$


is injective. Here $H_{\rho}^{*}$ denotes the $\rho$-equivariant cohomology with coefficients as in Theorem 1.

Proof. By Proposition 3, $N_{f}$ is a normally hyperbolic submanifold of $O_{f} \subset$ $M_{f}$. It is therefore by Proposition 1 of [3] a *-hyperbolic invariant set of the flow $\chi_{f}$ in $M_{f}$ (see [3, Definition 4]). We now construct a homotopy retraction from $M_{f}$ to $N_{f}$. Let us therefore restrict our attention to a continuous family $f_{\tau}, \tau \in \mathbb{R}$, with $f_{0}=f$. Set

$$
M_{\mathbb{R}}=p_{\mathbb{R}}(M \times \mathbb{R} \times(-1,1))
$$

where $p_{\mathbb{R}}$ is uniquely defined by

$$
p_{\mathbb{R}}(x, \tau, t)=p_{\mathbb{R}}\left(f_{\tau}(x), \tau, t+1\right),
$$

for $t \in(-1,0)$. There is a continuous map

$$
H: \mathbb{R} \times M \times[0,1] \rightarrow M:(\tau, x, t) \mapsto H_{\tau}(x, t)
$$

such that $H_{\tau}(x, 0)=r\left(f_{\tau}(x)\right)$ and $H_{\tau}(x, 1)=f(r(x))$. Define for $t \in[0,1]$

$$
r_{\mathbb{R}}\left(p_{\mathbb{R}}(x, \tau, t)\right)=p_{f}\left(H_{\tau}(x, t), t\right) .
$$

This defines a continuous map $r_{\mathbb{R}}: M_{\mathbb{R}} \rightarrow N_{f}$, since

$$
\begin{aligned}
r_{\mathbb{R}}\left(p_{\mathbb{R}}(x, \tau, 0)\right) & =p_{f}\left(H_{\tau}(x, 0), 0\right)=p_{f}\left(r\left(f_{\tau}(x)\right), 0\right) \\
& =p_{f}\left(f\left(r\left(f_{\tau}(x)\right)\right), 1\right)=p_{f}\left(H_{\tau}\left(f_{\tau}(x), 1\right), 1\right) \\
& =r_{\mathbb{R}}\left(f_{\tau}(x), \tau, 1\right) .
\end{aligned}
$$

The following lemma summarizes the properties of this construction.

Lemma 4.1. If $f: X \rightarrow X$ is a homeomorphism, then there exists an exact sequence

$$
H^{*}\left(X_{f}\right) \rightarrow H^{*}(X) \stackrel{f^{*}-\text { id }}{\longrightarrow} H^{*}(X) \stackrel{\delta}{\longrightarrow} H^{*+1}\left(X_{f}\right) .
$$

Moreover, if $r: X \rightarrow Y$ is a map which homotopy commutes with $f: X \rightarrow X$, $Y \rightarrow Y$, and $r_{f}: X_{f} \rightarrow Y_{f}$ is defined by (4.5) restricted to the set $\{\tau=0\}$, then the following diagram commutes

$$
\begin{array}{cccc}
H^{*}\left(Y_{f}\right) & \longrightarrow H^{*}(Y) \stackrel{f^{*}-\mathrm{id}}{\longrightarrow} H^{*}(Y) \stackrel{\delta}{\longrightarrow} H^{*+1}\left(Y_{f}\right) \\
\downarrow_{r_{f}^{*}} & \downarrow f^{*} r^{*} & \mid f^{*} r^{*} & \\
H^{*}\left(X_{f}\right) & \longrightarrow r_{f}^{*} \\
H^{*}(X) \stackrel{f^{*}-\text { id }}{\longrightarrow} H^{*}(X) \stackrel{\delta}{\longrightarrow} H^{*+1}\left(X_{f}\right) .
\end{array}
$$

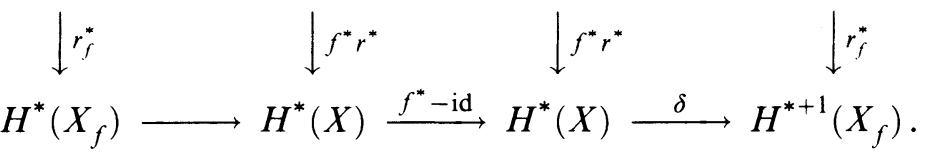

Proof. We consider the Mayer Vietoris sequence (see [9]) for

$$
X_{f}=p_{f}\left(X \times\left[0, \frac{1}{2}\right]\right) \cup p_{f}\left(X \times\left[\frac{1}{2}, 1\right]\right)
$$

which is given by

$$
\begin{aligned}
\stackrel{\delta}{\rightarrow} H^{*}\left(X_{f}\right) \rightarrow & H^{*}\left(p_{f}\left(X \times\left[0, \frac{1}{2}\right]\right)\right) \oplus H^{*}\left(p_{f}\left(X \times\left[\frac{1}{2}, 1\right]\right)\right. \\
& \stackrel{i_{1}^{*}+i_{2}^{*}}{\longrightarrow} H^{*}\left(p_{f}(X \times\{0\})\right) \oplus H^{*}\left(p_{f}\left(X \times\left\{\frac{1}{2}\right\}\right)\right) \stackrel{\delta}{\longrightarrow} .
\end{aligned}
$$


Applying the homotopy equivalence $\pi_{X} \circ p_{f}^{-1}$, we obtain the exact sequence

$$
H^{*}\left(X_{f}\right) \rightarrow\left(H^{*}(X)\right)^{2} \stackrel{\theta}{\longrightarrow}\left(H^{*}(X)\right)^{2} \longrightarrow H^{*+1}\left(X_{f}\right)
$$

with $\theta(\alpha, \beta)=\left(f^{*} \alpha+\beta, \alpha+\beta\right)$. Now define the isomorphism $j_{1}(x, y)=$ $(x-y, y)$ and $j_{2}(\gamma, \delta)=(\gamma, \delta-\gamma)$ of $\left(H^{*}(X)\right)^{2}$. Since

$$
j_{1} \circ \theta \circ j_{2}(\alpha, \beta)=\left(f^{*} \alpha-\alpha, \beta\right),
$$

we can eliminate the factor $H^{*}(X)$ in (4.11) and obtain the sequence (4.7).

If we set up the same sequence for $X$ replaced by $Y$, then the maps $R_{f}^{*}$ : $H^{*}\left(Y_{f}\right) \rightarrow H^{*}\left(X_{f}\right)$ and $f^{*} r^{*} \oplus f^{*} r^{*}:\left(H^{*}(Y)\right)^{2} \rightarrow\left(H^{*}(X)\right)^{2}$ commute with the exact sequences. Moreover, the latter homomorphism commutes with $j_{1}$ and $j_{2}$, as one readily verifies. Hence eliminating one factor $H^{*}(X)$ as before, we obtain the commuting diagram (4.8).

Setting $X=Y=N$ in (4.8), we conclude by the five lemma (see [9]) that $f_{f}: M_{f} \rightarrow N_{f}$ induces isomorphisms in cohomology when restricted to $N_{f}$. Now the proof of [3, Theorem 2] applies to this situation and we can conclude that under the hypothesis of Theorem 3,

$$
\left(r_{f} \mid T_{f}\right)^{*}: H_{\rho}^{*}\left(N_{f}\right) \rightarrow H_{\rho}^{*}\left(T_{f}\right)
$$

is injective. In fact, although Theorem 2 of [3] formally requires $r_{f}$ to be a retraction, the proof depends only on the fact that $\left(r_{f} \mid N_{f}\right)^{*}$ is injective.

We now have to pass from $T_{f}$ to the fibre $T$. Note that in order to prove the injectivity of $(4.1)$, it suffices to prove that

$$
r^{*}[N] \neq 0,
$$

where $[N] \in H_{\rho}^{d}(N), d=\operatorname{dim} N$, is the fundamental class. In fact, it then follows from Poincaré duality (see [9]) that for every $x \in H_{\rho}^{*}(N), x \neq 0$, there exists a class $y \in H_{\rho}^{*}(N)$ with $x \cup y=[N]$. Then $\left(r^{*} x\right) \cup\left(r^{*} y\right)=r^{*}[N] \neq 0$ implies that $r^{*} x \neq 0$.

To prove (4.14), we apply (4.8) of Lemma 4.1 to $X=N$ and $Y=T$. Since $f: N \rightarrow N$ is a diffeomorphism, we conclude that for coefficients as in Theorem $1, f^{*}[N]=[N]$. Therefore, $f^{*}-\mathrm{id}$, so that $\delta[N] \neq 0$. Hence $r_{f}^{*} \delta[N] \neq 0$ by injectivity of (4.13). Since (4.8) is commutative, we also have $\delta \circ f^{*} \circ r^{*}[N] \neq 0$, which proves $(4.14)$. This completes the proof of Theorem 3.

In order to obtain a perturbation result, choose a ( $\rho$-invariant $)$ neighborhood $U$ of $N$ in $M$ with a ( $\rho$-invariant) retraction $r$. For example, we can use the metric $g$ on $M$ defined in $\S 3$ to define a diffeomorphism of the disc bundle $D_{\varepsilon} \subset E^{+} \oplus E^{-}$onto $U:=\exp _{g}\left(D_{\varepsilon}\right)$ by means of

$$
\exp _{g}: D_{\varepsilon} \rightarrow N \text {. }
$$

We can define a retraction $r$ which corresponds to the bundle projection $D_{\varepsilon} \rightarrow N$ under $\exp _{g}$. It is homotopic to $\left.f^{-1}\right|_{N} \circ r \circ f$, since all retractions 
$D_{\varepsilon} \rightarrow N$ are homotopic to the projection as is easily verified. Choosing such a homotopy $H$, we can define $r_{f}$ as in (4.5). Now we apply Theorem 2 of [3] to the flow on the open set $U_{f}=p_{f}(U \times(-1,1)) \subset M_{f}$.

Moreover, it is known that if $U_{f}$ is isolating for flow $\chi_{f}$, then this is true for all $f_{\lambda}$ for $\lambda$ small enough as in Theorem 1. For example, this is shown in [1, III.3]. In this case, $U$ is also isolating for $f^{\prime}$ by Proposition 2. We can now apply Theorem 2 to the manifold $U$ and the retraction $r$ in order to conclude that the maximal invariant set $T=T_{f^{\prime}}(U)$ satisfies the assertion of Theorem 1. This completes the proof of Theorem 1 .

\section{REFERENCES}

1. C. D. Conley, Isolated invariant sets and the Morse index, CBMS Regional Conf. Ser. in Math., no. 38, Amer. Math. Soc., Providence, R. I., 1978.

2. N. Fenichel, Persistence and smoothness of invariant manifolds for flows, Indiana Univ. Math. J. 21 (1971), 193-226.

3. A. Floer, $A$ refinement of the Conley index and an application to the stability of hyperbolic invariant sets, Ergodic Theory and Dynamical Systems 7 (1987), 93-103.

4. J. K. Hale, Ordinary differential equations, Wiley, New York, 1969.

5. S. Helgason, Differential geometry, Lie groups, and symmetric spaces, Academic Press, New York, 1978.

6. M. W. Hirsch, C. C. Pugh and M. Shub, Invariant manifolds, Lectures Notes in Math., vol. 583, Springer-Verlag, 1977.

7. J. Jarik and J. Kurzweil, On invariant sets and invariant manifolds of differential systems, J. Differential Equations 6 (1969), 247-263.

8. J. McCarthy, Stability of invariant manifolds, Bull. Amer. Math. Soc. 61 (1955), 149-150.

9. E. Spanier, Algebraic topology, McGwraw-Hill, New York, 1966.

Department of Mathematics, University of California, Berkeley, California 94720 